\title{
Aforismos sobre Enfermedad Pulmonar Obstructiva Crónica e Insuficiencia Cardíaca
}

\section{Aphorisms about Chronic Obstructive Pulmonary Disease and Heart Failure}

\author{
Julio Montes ${ }^{1,2}$, Fernando de la Iglesia ${ }^{1,2}$, Emilio Casariego $0^{1,2}$ \\ José Manuel Cerqueiro², José Alberto Fernández-Villar³, Jaime Gonzálvez-Rey4 ${ }^{4}$, José Luis Jiménez², \\ Esperanza Moldes ${ }^{4}$, Carlos Moral ${ }^{4}$, Pilar Taladriz Cobas ${ }^{5}$, Rosario Timiraos Carrasco ${ }^{4}$, Alfonso Varela ${ }^{6}$, Carmen Varela . \\ ${ }^{1}$ Coordinador, ${ }^{2}$ Internista, ${ }^{3}$ Neumólogo, ${ }^{4}$ Médico de Familia, ${ }^{5}$ Enfermera, ${ }^{6}$ Cardiólogo.
}

\section{Justificación}

En el año 2012 la Sociedad Gallega de Medicina interna (SOGAMI) elaboró un número monográfico de Atención al Paciente crónico y con pluripatología'. Se inscribía en la filosofía de la Declaración de Sevilla (2011) de atención al paciente de estas características². En esta se definía como esencial la implicación de todos los recursos de la comunidad en la asistencia a tales pacientes y se destacaba el importante papel de las Sociedades científicas. Para ello en Galicia, la SOGAMI promovió un plan de comienzo para informar e involucrar a sus miembros en una estrategia racional y eficiente en el manejo de pacientes crónicos y pluripatológicos. Con este objetivo en dicho monográfico se establecía un plan de ruta con pasos sucesivos que incluían:

1. Conocimiento del punto de partida y divulgación de los planes ya en desarrollo en distintos ámbitos asistenciales del SERGAS.

2. Plasmar dichas experiencias multicéntricas en un Monográfico de Galicia Clínica, revista oficial de la SOGA$\mathrm{Ml}$, y difundirlas a través de su Web (www.meiga.info)3. Ello se consiguió en gran parte con la publicación del Monográfico mencionado, disponible en la web, y que se distribuyó inicialmente el IV Congreso Nacional de Atención Sanitaria al Paciente Crónico de Atención al Paciente Crónico (Alicante, 2012) y también se ha venido distribuyendo entre los miembros de la SOGAMI y otros foros de Atención a dicho paciente, singularmente los Médicos de Atención Primaria, merced al patrocinio no condicionado de AstraZéneca.

3. El tercer paso consiste en la elaboración o adaptación de Guías de manejo de pacientes pluripatológicos para proporcionar cuidados integrados y en lo posible homogéneos a estos pacientes, incluyendo prioritariamente aquellas patologías más prevalentes como Enfermedad Pulmonar Obstructiva Crónica (EPOC) e insuficiencia cardiaca (IC), dislipemias, HTA, etc. Ello se seguiría de la divulgación e implementación de estas directrices entre Ios miembros de la SOGAMI y Sociedades afines a tra- vés de su Revista y página Web específicas así como de otros medios de difusión y su adaptación a los diferentes ámbitos locales ${ }^{3}$.

\section{Metodología y pasos de elaboración}

Este documento, constituye la plasmación de este intento de elaboración de directrices de manejo de pacientes pluripatológicos con IC y EPOC.

Para ello, se identificaron e invitó personalmente a un grupo multidisciplinar de expertos en tales temas con representación de Medicina interna, Atención Primaria, Cardiología, Neumología y Enfermería. Se formaron dos equipos. Uno se centró en IC y debía evaluar su repercusión sobre EPOC y otro centrado en EPOC que estudiara la repercusión sobre IC. Cada equipo constó de 5 miembros (un internista, dos médicos de Atención primaria, un especialista (Cardiología o Neumología) y un diplomado en enfermería con experiencia en insuficiencia cardiaca o EPOC, dirigidos ambos por un coordinador internista. A ellos se incorporó un Experto en Metodología de Elaboración de Guías y Protocolos clínicos.

Se actuó de acuerdo al siguiente proceso en fases:

$1^{\text {a }}$ Fase. Reclutamiento y confección definitiva de equipos. Se contactó personalmente y telefónicamente con reconocidos expertos de las Sociedades mencionadas sobre los temas tratados, intentando que se incorporaran miembros procedentes de todas las provincias gallegas.

$2^{\mathrm{a}}$ Fase. Desarrollo de metodología. Para ello se celebró una Reunión de trabajo en Santiago de Compostela (29 de noviembre 2012) en la cual el Dr. Emilio Casariego, experto en Metodología y elaboración de Guías Clínicas (encargado por ejemplo del Grupo de Medicina Basada en la Evidencia y de metodología de elaboración y evaluación de Guías para Fisterra.com) presentó en una sesión de 3 horas los objetivos y métodos de realización. En dicha sesión se constituyeron definitivamente los grupos y se fijaron las tareas y objetivos para el periodo siguiente.

$3^{\text {a }}$ Fase. Documentación. Explicada dicha metodología, se encargó a una Documentalista experta (Srta. A. Blanco) la 
labor de recopilar y clasificar el material relevante al respecto. Este fue enviado a los miembros de los diversos equipos. $4^{a}$ Fase. Trabajo de equipos en ambas patologías. Con dicha documentación los equipos trabajaron por separado para clasificar y comentar la bibliografía identificada relevante (tanto la remitida como aquella ulterior identificada por el trabajo de cada miembro). Esta fue mandada con los comentarios relevantes pertinentes a los coordinadores. En esta fase cada coordinador y su grupo mantuvieron contacto por correo electrónico. Se evaluaron y se clasificaron así más de 200 documentos relevantes sobre tales temas y se realizó una primera síntesis de los mismos.

$5^{a}$ Fase. Documento provisional. En una reunión de los coordinadores con el responsable de Metodología y ante la constatación de la ausencia de evidencias firmes en este campo se decidió que las recomendaciones elaboradas adoptarían la forma de aforismos, que tuvieran carácter práctico, y de la mayor utilidad posible. Sólo para ilustrar tal dificultad en este campo de la elaboración de recomendaciones señalar que un grupo números de Expertos de la American Thoracic Society (ATS) y la European Respiratory Society (ERS) vienen elaborando una Guía sobre EPOC y sus comorbilidades, habiendo publicado un posicionamiento en la revista Lancet ${ }^{4}$ y cerca de 14 artículos sucesivos en 2012 y 2013 sólo sobre los aspectos metodológicos que debería reunir dicha Guía ${ }^{5}$.

$6^{\text {a }}$ Fase. El coordinador de un grupo remitió las conclusiones al responsable del otro Grupo. Este elaboró un tríptico con los aforismos sobre dichas comorbilidades. Se consideró también la utilidad, como recordatorio práctico, de incluir las recomendaciones diagnósticas y de tratamiento de cada entidad por separado (EPOC e IC) según las Guías más acreditadas en tales campos (Guías de la European Society of Cardiology 2012 para IC y Guía GESEPOC 2012 para EPOC).

Este tríptico se remitió por correo electrónico a los miembros de los grupos de trabajo para que formularan sus correcciones y sugerencias.

Un avance preliminar de este tríptico pudo hacerse en el V Congreso Nacional de Atención Sanitaria al Paciente Crónico (Barcelona, abril 2012).

Se espera poder hacer una presentación parcial de estos aforismos en el Congreso de la SOGAMI (Lugo, junio 2013). Se ha contactado con Astra-Zeneca para evaluar la posibilidad de que con su patrocinio no condicionado pueda imprimirse tal tríptico.

A continuación se detallan tales recomendaciones, omitiendo las referidas a cada entidad por separado. Se omite, salvo alguna excepción, y para evitar farragosidades, la bibliografía que se incorporará también al tríptico y publicación completa en página web. Veáse actualización de este apartado en: www.meiga.info/aforismosEPOC-IC.

\section{Bibliografía}

1. Montes Santiago J, Casariego Vales E, coord. La atención al paciente crónico y pluripatológico. El caso de Galicia. Gal Clin. 2012; 73 (Supl. 1); S1-S47.

2. Ollero Baturone M, Orozco Beltrán, D, Domingo Rico C et al. "Declaración de Sevilla" conferencia nacional para la atención al paciente con enfermedades crónicas. Rev Clin Esp. 2011; 211: 604-6.

3. Montes Santiago J, Casariego Vales E, De Toros Santos M, Mosquera E. La asistencia a pacientes crónicos y pluripatológicos. Magnitud e iniciativas para su manejo: La declaración de Sevilla. Situación y propuestas en Galicia. Gal Clin. 2012; 73 (Supl. 1): S7-S14.

4. Schünemann HJ, Woodhead M, Anzueto A, et al. A vision statement on guideline development for respiratory disease: the example of COPD. Lancet. 2009; 373: 774-9.

5. Wilson KC, Irwin RS, File TM Jr, et al.; ATS/ERS Ad Hoc Committee on Integrating and Coordinating Efforts in COPD Guideline Development. Reporting and publishing guidelines: article 12 in Integrating and coordinating efforts in COPD guideline development. An official ATS/ERS workshop report. Proc Am Thorac Soc. 2012, 9:293-7.

\section{DIAGNÓSTICO}

1. En España $\approx 1 / 3$ de EPOC presentan IC $y \approx 1 / 3$ de IC presentan EPOC

$\rightarrow$ Dificultad/retraso de diagnóstico: $\uparrow$ mortalidad y hospitalizaciones de ambas.

2. En presencia EPOC+IC se realizan menos test de confirmación para IC y EPOC

En diagnóstico de IC se necesita ecocardiografía (Guías ESC, 2012).

En diagnóstico de EPOC se necesita espirometría (Guías GOLD, GESEPOC)

IC + EPOC presentan obstrucción combinada/restricción en PFR.

Con diuresis $\rightarrow \mathrm{FEV} 1 \uparrow 35 \%$. Para dco. de EPOC $\rightarrow$ posponer espirometría hasta estabilización

Con = obstrucción ( $\approx F E V 1)$ un EPOC+IC se clasificará > grave que EPOC sin IC y viceversa, porque los tests de función cardiopulmonar están significativamente afectados.

3. BNP/NT-proBNP $\rightarrow$ útiles en afirmar/descartar IC en presencia de EPOC.

$\rightarrow$ BNP + juicio clínico=95-100\% de certeza de diagnóstico.

También útiles en EPOC con disnea $\rightarrow \downarrow$ estancia y coste.

4. La determinación de BNP puede no ser tan útil para definir causa de disnea en: ancianos y/o comorbilidades que modifican sus niveles en plasma (anemia, I. renal).

5. Considerar ICFEP si FEVI $>40 \%$.

Valorar $\uparrow \mathrm{VI}, 0 \uparrow A \mathrm{Al}$ en ecocardio.

$\rightarrow$ Valorar Respuesta a IECAS y diuréticos de asa.

\section{TRATAMIENTO}

Fármacos en EPOC $\rightarrow$ en IC $\quad$ Fármacos en IC $\rightarrow$ en EPOC

Agonistas B2-adrenérgicos

Agonistas muscarínicos

Betabloqueantes

Corticoides inhalados

Corticoides orales

Metilxantinas
Estatinas

IECAS/ARA ||

Diuréticos 
Fig. 1. Aproximación diagnóstica utilizando BNP/NT-proBNP.

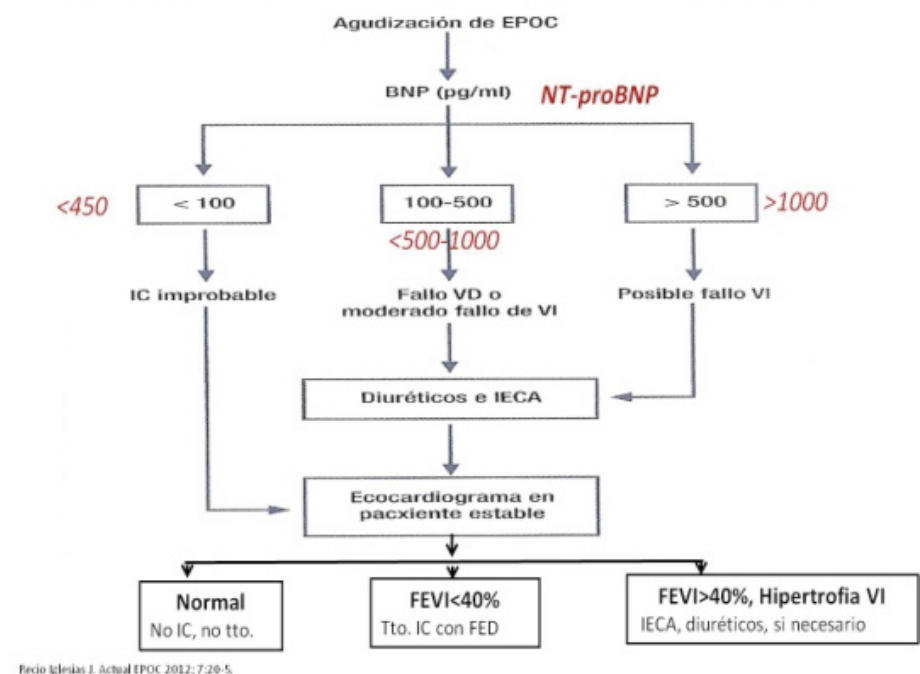

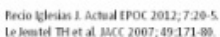

EPOC en pacientes con ICFED se asocia a $\downarrow$ uso de terapias útiles para esta (betabloqueantes, IECAs, antag. aldosterona) y muestra tendencia a peor pronóstico.

\subsection{Fármacos en EPOC sobre IC}

\section{A) Agonistas B2 adrenérgicos}

\section{Consideración general.}

Difícil deslindar efectos de drogas o de enfermedad. Estudios pequeños, contradictorios.

2. Incidencia de IC. Estudios contradictorios sobre $\uparrow 0 \approx$ en general o neumopatías.

$\rightarrow$ No claras recomendaciones específicas, pero evitar vía oral.

3. Uso en nebulización. Dosis $10 \times$ que tradicionales.

Pueden producir arritmias, isquemia cardiaca y descompensación de IC. Otros estudios no documentan efectos desfavorables en IC.

$\rightarrow$ Evitarlos en descompensación si sospecha de $\mathrm{EPOC}+\mathrm{IC}$.

4. Uso en vía inhalatoria. Contradictorio.

A) $\uparrow$ Morbimortalidad. Au AD et al. Chest 2003; 123 : 1964): En 1529 p. con ICFED la mortalidad e ingresos por IC $\rightarrow n^{0}$ de envases/mes a partir de 2 envases/mes).

Estudio ACQIP (Au AD et al. Am Heart J 2004, 148: 915): Beta estimulantes $\rightarrow$ 个ingresos por IC (OR:1,8). Efecto perdido al añadir otros factores [tabaquismo, edad, enfermedades cardiovasculares asociadas, corticoides] por lo que tales efectos adversos $\rightarrow$ gravedad de la enfermedad subyacente.

Estudio CHARM (broncodilatadores $\rightarrow \uparrow$ mortalidad global, mortalidad cardiovascular e ingresos. No distingue broncodilatador. $\rightarrow$ No posible si por drogas, y/o gravedad EPOC
B) No efecto sobre mortalidad. Estudio TORCH (McGarvey LP Thorax 2007; 62. 411); RS (Rodrigo GJ, Chest 2008; 133: 1079).

El salmeterol en vía inhalatoria en IC grado $\|/\| I \rightarrow \uparrow F E V 1$ (6\%)

Recomendación clínica. Uso aislado o combinación según GESEPOC/GOLD.

No hay estudios prospectivos de B2 adrenérgicos en IC aguda 0 descompensada.

5. Interacciones entre betabloqueantes-beta2adrenérgicos.

Probablemente esta interacción influya pero no existen evidencias claras.

Pérdida de beneficio de betabloqueantes en caso de infarto de miocardio.

Recomendación clínica. Los bbloqueantes mejoran síntomas y supervivencia en IC y también EPOC. No debe omitirse tal terapia en IC (Grado: IA).

EPOC avanzada: no contraindicación. Única contraindicación: Asma grave cierta.

Se recomienda comenzar por la dosis más pequeña y monitorización de síntomas.

Añadir un agente antimuscarínico si se necesita broncodilatación.

Debe hacerse los controles de FC previa a la inhalación de aerosoles.

Preferible el uso de dispositivos de polvo seco por mejor adherencia.

Indacaterol. Seguridad cardiovascular de indacaterol es $\approx$ placebo $\mathrm{y} \approx$ otros $\mathrm{BD}$ de larga duración (formoterol, salmeterol, tiotropio).

\section{B) Anticolinérgicos.}

1. En general seguros, pero datos contradictorios.

RS (Singh et al, JAMA 2008; 300: 1439) $\rightarrow$ 个riesgo CV. Estudio UPLIFT (Tashkin DP et la NEJM 2008; 359: 1543) $\rightarrow \downarrow I C$ y IAM.

Ipratropio $\rightarrow$ seguro en IC.

Tiotropio $\rightarrow$ mejora calidad de vida, $\downarrow$ exacerbaciones y reingresos. No diferencias en ingresos por cualquier causa y mortalidad.

La administración de tiotropio mediante el sistema Respimat inhaler, que se comunicó asociada a $\uparrow$ mortalidad CV en estudios poblacionales (Verhamme KM et al, Eur Respir J. 2013; 42:606-15), se ha demostrado segura y equivalente a la de HandiHaler en el muy reciente estudio TIOSPIR (N Engl J Med 2013; DOI: 10.1056/NEJMoa1303342) y por ello puede recomendarse con seguridad su uso (Jenkins CR. More Than Just Reassurance on Tiotropium Safety. N Engl J Med 2013. D0l:10.1056/ NEJMe1310107). 


\section{C1) Corticoides inhalados}

En EPOC $\rightarrow$ parecen $\downarrow$ riesgo cardiovascular (Estudio EUROSCOP, TORCH).

Sin embargo, debido a la incidencia de otros efectos secundarios ( $p$. ej. aumento de neumonía) deben utilizarse en exacerbaciones siempre asociados a betadrenérgicos y según recomendaciones GOLD o GESEPOC (Gravedad III 0 superiores).

\subsection{Fármacos en IC sobre EPOC}

\section{A. Betabloqueantes.}

En casi todos los estudios con Betabloqueantes en IC se excluye EPOC.

Se infrautilizan en pacientes con EPOC e IC. Especialmente indicados si coexiste HTA o enfermedad cardiovascular.

En IC y EPOC estable no contraindicado uso de Betabloqueantes (GR: 1, NE: B).

Única contraindicación: antecedentes de asma grave. Inicio a bajas dosis y aumento gradual. Los deterioros ligeros de función pulmonar y síntomas leves no deben llevar a abandonarlos prematuramente.

Aunque se recomiendan Betabloqueantes selectivos (bisoprolol, nevibolol o atenolol), no hay datos concluyentes sobre el beneficio real de cardioselectividad.

Estudios OPTIMIZE-HF, VALHEFT o VALIANT (este con IAM) 0 estudios retrospectivos, en el cual amplios porcentajes tenían EPOC $\rightarrow$ bbloqueantes presentaron $\uparrow$ supervivencia, independientemente de cardioselectividad. Carvedilol (no selectivo) bien tolerado en EPOC.

Bisoprolol en IC + EPOC moderada-severa $\downarrow$ FEV1 que metoprolol pero sin efecto clínico aparente.

Recomendación clínica. Los betabloqueantes, incluso los no cardioselectivos, son bien tolerados y seguros en pacientes con IC + EPOC.

\section{B) Diuréticos}

Precaución con diuréticos de asa $\rightarrow$ alcalosis metabólica, $\uparrow \mathrm{pCO2}$ y agrava hipoventilación.

\section{C) Estatinas, IECA y ARA-II}

Recomendación clínica. Mejoran morbimortalidad en IC y también en EPOC.

Además disminuyen incidencia de IAM (independientemente de esteroides).

Considerar ARA-II sobre IECAS por disminuir incidencia de tos.

\section{RECOMENDACIONES EN SEGUIMIENTO}

\section{Generales.}

Abandono del hábito tabáquico.

Vacunación antigripal anual y antineumocócica.

Atención a polifarmacia

Atención a comorbilidades frecuentes como diabetes mellitus, insuficiencia renal.
Evitar glitazonas en IC. Evitar metformina en IR avanzada. Artrosis/Artritis. AINES están contraindicados en IC.

El dolor puede requerir opioides suaves.

Despistaje y tratamiento de la depresión. El tratamiento de la disnea puede aliviarla.

Evitar antidepresivos tricíclicos en la IC.

Despistaje y tratamiento de síndrome de apnea-hipopnea del sueño.

Embolismo pulmonar $\rightarrow$ profilaxis sistemática cuando apropiado.

2. La rehabilitación (programa de entrenamiento) en EPOC y comorbilidades, incluyendo cardiovasculares $\rightarrow \downarrow$ secuelas de agudizaciones mejorando calidad de vida y capacidad de esfuerzo.

3. Los programas de autocuidado en pacientes con EPOC e IC $\rightarrow \uparrow$ calidad de vida y capacidad de ejercicio, incluso tras finalización del programa.

4. Las unidades multidisciplinares de IC $\rightarrow \downarrow$ hospitalizaciones (Grado IA).

5. La telemonitorización domiciliaria $\rightarrow \downarrow$ mortalidad en IC y $\downarrow$ hospitalización por cualquier causa en pacientes con EPOC.

Aún $\rightarrow$ insuficiente evidencia de beneficio (Bolton CE et al. J Eval Clin Pract. 2011;17: 1216-22).

6. La atención domiciliaria en EPOC después de un alta hospitalaria $\rightarrow$ no $\downarrow n^{0}$ de veces que acudieron al hospital ni readmisiones, pero sí sus conocimientos sobre la enfermedad y ciertos aspectos de calidad de vida.

Otras recomendaciones de evidencia grado I (Arnold, 2006, Mutasingwa, 2011):

1. El anciano con sospecha y/o diagnóstico de IC se evaluará para comorbilidades que influyan en el tratamiento, adherencia, seguimiento y pronóstico.

2. En anciano con IC hospitalizado se evaluará delirio cuando sea apropiado.

3. El anciano frágil con IC y alta comorbilidad será seguido con un programa de seguimiento de enfermedad.

4. El Médico de Atención Primaria y cuidadores estarán involucrados en planificación y seguimiento del anciano frágil con IC.

5. Deben evaluarse los aspectos psicosociales (por ejemplo depresión, soledad, miedo, apoyos familiares y en casa...) y reevaluados periódicamente.

6. Los cuidadores de pacientes con IC avanzada serán evaluados sobre el cuidado proporcionado y la carga de estos.

\section{Bibliografía}

1. Arnold JM, Liu P, Demers $C$ et al. Canadian Cardiovascular Society consensus conference recommendations on heart failure 2006: diagnosis and management. Can J Cardiol 2006; 22 (1): 23-45.

2. Mutasingwa DR, Hong Ge H, Upshur REG. How applicable are clinical practice guidelines to elderly patients with comorbidities? Can Fam Physician 2011; 57: e253-62. 